\title{
Early use of anti-TNF agents might give a helping hand to paediatric patients with Crohn's disease
}

I nitial decisions concerning the early treatment of paediatric patients with Crohn's disease are of crucial importance, both to promote the best possible quality of life in these patients and to limit any potential drug-related effects on their subsequent growth development. "If we knew who was likely to do poorly and who was likely to do well at diagnosis, it would certainly help to direct our therapies," states Jeffrey Hyams, corresponding author of a new study investigating this issue, now published in Gastroenterology.

Immunomodulators (most commonly thiopurines) are the traditional early treatment for patients with moderateto-severe Crohn's disease. However, whether these agents alter the natural history of the disease or affect children's growth potential remains unclear. The development of anti-TNF agents was an important advance in the medical management of paediatric Crohn's disease, yet the optimal position of these agents in therapeutic algorithms for paediatric patients is still poorly defined.

Hyams and colleagues hypothesized that early anti-TNF therapy, given within the first 3 months of diagnosis of Crohn's disease, would produce better outcomes at 1 year in paediatric patients than early use of immunomodulators. To investigate this hypothesis, the researchers conducted a comparative effectiveness study to specifically analyse the efficacy of these different therapeutic approaches for treating disease of similar severity. Of note, anti-TNF agents have already been shown to have superior efficacy in randomized controlled trials in patients with a range of disease severity.

Patients were identified from the RISK project, an observational research programme sponsored by the Crohn's and Colitis Foundation of America that is investigating the clinical, serological and genetic predictors of complicated disease in children with newly diagnosed Crohn's disease. The RISK cohort comprises 552 patients (aged $<17$ years) with Crohn's disease from paediatric gastroenterology centres in North America.

Using propensity score methodology, the researchers identified 204 paediatric patients (median age 11.8 years) from the RISK cohort who had matched baseline characteristics and who had received early anti-TNF therapy, early immunomodulators or no early immunotherapy. The relationships between the different therapies and surgery-free remission and growth at 1 year were then investigated for these selected patients.

The researchers found that early treatment with an anti-TNF agent was superior to early treatment with an immunomodulator ( $85.3 \%$ versus $60.3 \%$ in remission at 1 year, respectively; $P=0.0017)$. Of note, no statistically significant difference was found in the number of patients in remission at 1 year between those who had received early immunomodulator therapy and those who had received no early immunotherapy ( $60.3 \%$ versus $54.4 \%$; $P=0.49$ ). AntiTNF agents remained superior to immunomodulators when therapy was provided for $>3$ months.

In terms of growth potential-an important outcome when investigating the effectiveness of treatments in paediatric patients-weight and BMI z scores increased in all three patient groups. However, the mean height $\mathrm{z}$-score only showed improvement when compared with baseline in patients who received early treatment with anti-TNF agents.

Overall, the researchers conclude that in children who are newly diagnosed with comparably severe Crohn's disease, early monotherapy with anti-TNF agents improves clinical and growth outcomes at 1 year compared with monotherapy with an immunomodulator.

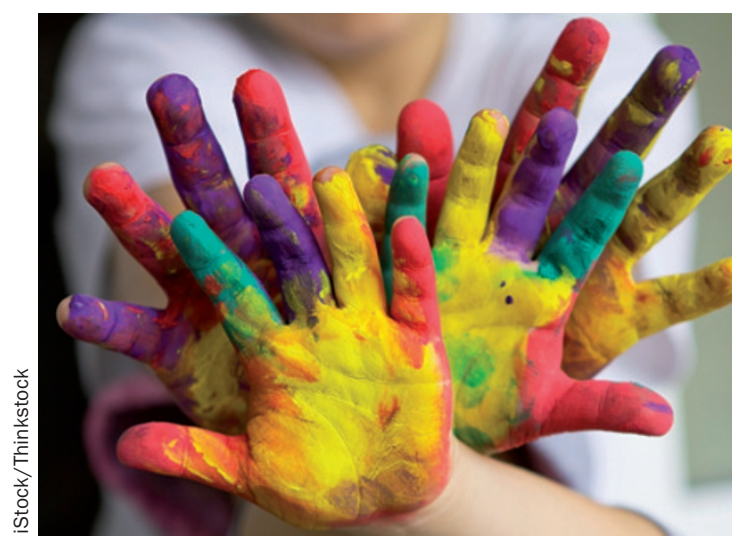

Salvatore Cucchiara, an expert in the field who was not involved in this study, notes that the dosing of the agents was not protocol-based, but was determined by the attending physician. He also states that "we need a randomized prospective blinded protocol to assess if early introduction of biologics in paediatric Crohn's disease can influence long-term clinical course. This study should include objective assessment of mucosal healing and imaging evaluation of structural damage”.

These data provide a guiding hand in selecting treatments early after diagnosis for paediatric patients with Crohn's disease. "However, we were not able to identify any particular clinical feature that identified which subset of children this [approach] might be more appropriate for," Hyams cautions. Looking to the future, the researchers "will further analyse our cohort looking at serology and genetics to see if these factors can be identified".

Katherine Smith 